ISSN 1420-3049

www.mdpi.com/journal/molecules

Article

\title{
Synthesis with Nitriles: Synthesis of Some New Mercapto- pyridazine, Mercaptopyridazino[1,6-a]quinazoline and Thiophene Derivatives
}

\author{
Mariam A. Al-Sheikh \\ Chemistry, Girls College of Education, Jeddah, P. O. Box 138016, Jeddah 21323, Kingdom of Saudi \\ Arabia; E-mail: r1425@hotmail.com
}

Received: 17 July 2008; in revised form: 20 October 2008 / Accepted: 28 October 2008 / Published: 4 November 2008

\begin{abstract}
Bromophenyl)-2-thiocyanatoethylidene)malononitrile (3) undergoes azo coupling with diazotized aromatic amines to afford arylhydrazone derivatives, which are readily cyclized to afford the corresponding $3(2 H)$-pyridazinimine derivatives upon reflux in aqueous $\mathrm{NaOH}$. Under similar condition an $o$-cyanoarylhydrazone derivative was cyclized into 6H-pyridazino[1,6-a]quinazolin-6-imine, which in turn was easily transformed into $6 \mathrm{H}$-pyridazino[1,6-a]quinazolin-6-one on reflux in ethanolic/ $\mathrm{HCl}$. Compound 3 afforded substituted 5-acetylthiophene derivatives upon reflux in $\mathrm{AcOH} / \mathrm{HCl}$ mixtures.
\end{abstract}

Keywords: Thiophene; Pyridazine; Pyridazino[1,6- $a$ ]quinazoline.

\section{Introduction}

During the past few decades there has been increasing interest in the synthesis and properties of pyridazines, pyridazinones and pyridopyridazinones. Pyridazines and pyridazino[1,6- $a$ ]quinazolines show diuretic [1], antihyprertensive [2,3], anticonvulsant, antispasmodic and muscle relaxant activities $[3,4]$. They inhibit blood platelet aggregation [5] and are active in the treatment of diabetic complications [6]. In addition, these compounds have been tested as cardiac [7] and tuberculostatic agents, as fungicides [4] and as herbicides [8]. Their use as antiasthmatics, analgesics and inflammation inhibitors has also claimed [9]. Recently, the pyridazinone nucleus has been extensively 
studied in a variety of medicinal agents [10], particularly as an important pharmacophore in the search for drugs acting on the cardiovascular system [11].

Continuing our interest in synthesis of pyridazines [12-14], some new functionally substituted pyridazine and pyridazinoquinazoline derivatives were required. 2-(1-(4-Bromophenyl)-2-thiocyanatoethylidene)malononitrile (3) seemed a good candidate to fulfil this objective via its coupling with the diazotized aromatic amines 4a-d to afford the arylhydrazone derivatives 5a-d, followed by cyclization to the pyridazines 6a-d (Scheme 1), by analogy with previously reported work on related systems [15, $16]$.

\section{Results and Discussion}

It has been found that reaction of compound $\mathbf{1}$ with potassium thiocyanate in ethanol produced the thiocyanate derivative $\mathbf{2}$ in $80 \%$ yield. Compound $\mathbf{2}$ condensed with malononitrile in ethanol in the presence of piperidine to afford the Knoevenagel condensation product 3 in $74 \%$ yield. Compound 3 undergoes azo coupling reaction with diazotized aromatic amines to afford the arylhydrazone derivatives 5a-g. Analytical and spectral data of these new arylhydrazone compounds were in complete agreement with the proposed structures. It had been previously reported [17] that similar systems could be cyclized in acidic media, however, in our hands prolonged reflux under such acidic conditions did not produce the desired pyridazine derivatives 6a-d, and we were only able to effect the cyclization of these arylhydrazone derivatives by refluxing in $20 \%$ ethanolic sodium hydroxide solution, although the SCN group was simultaneously hydrolysed to a SH group. Thus, compounds 5ad were cyclized to give the corresponding 3(2H)-pyridazinimine derivatives 6a-d, respectively (Scheme 1). This cyclization is assumed to proceed via the hydrazonothiol intermediate, and the other possibility of cyclization to give the thiophene derivatives 7a-d was readily ruled out on the basis of the ${ }^{1} \mathrm{H}-\mathrm{NMR}$ spectra of the products, which revealed the SH and $\mathrm{NH}$ signals at $\delta=6.81$ and $8.27 \mathrm{ppm}$, respectively, besides the aromatic protons at $7.52 \mathrm{ppm}$. In the case of compound $6 \mathbf{d}$, the ${ }^{13} \mathrm{C}-\mathrm{NMR}$ and mass spectra were also in agreement with the proposed structure.

The arylhydrazone derivatives $\mathbf{5 e}$ underwent a cyclization reaction under conditions similar to those used for compounds 5a-d to afford the 2-mercapto-6H-pyridazino[1,6- $a$ ]quinazolin-6-imine derivative 8a, which was assumed to result from a double internal Michael addition of the NH to the neighboring CN group. The IR spectrum of 8a showed a broad NH absorption band at 3435, 3324 and a CN absorption band at $2224 \mathrm{~cm}^{-1}$. Its ${ }^{1} \mathrm{H}-\mathrm{NMR}$ spectrum revealed two singlets at 6.88 and $8.31 \mathrm{ppm}$, which were attributed to the $\mathrm{SH}$ and $\mathrm{NH}$ protons, respectively. The aromatic protons appeared at $7.72 \mathrm{ppm}$. The elemental analysis of $\mathbf{8 a}$ was in good agreement with the proposed structure.

Compounds $\mathbf{5 f}$ and $\mathbf{5 g}$ underwent a similar cyclization under the same conditions to produce the 2mercapto-6H-pyridazino[1,6-a]quinazolin-6-one derivative $\mathbf{8 b}$, apparently via loss of water or methanol, respectively. The IR spectrum of $\mathbf{8 b}$ showed absorption bands at 2214 and $1674 \mathrm{~cm}^{-1}$ corresponding to $\mathrm{CN}$ and $\mathrm{C}=\mathrm{O}$ groups, respectively. The ${ }^{1} \mathrm{H}-\mathrm{NMR}$ spectrum of $\mathbf{8 b}$ revealed only one proton singlet at $6.80 \mathrm{ppm}$, which was attributed to the $\mathrm{SH}$ group, in addition to the aromatic protons at $8.23 \mathrm{ppm}$. Compound $\mathbf{8 b}$ could be obtained quantitatively from $\mathbf{8 a}$ upon refluxing the latter in ethanolic hydrochloric acid solution. The two products were matched by mixed m.p. and TLC analysis. 
Scheme 1. Reactivity of 2-(1-(4-bromophenyl)-2- thiocyanatoethylidene)malononitrile (3).

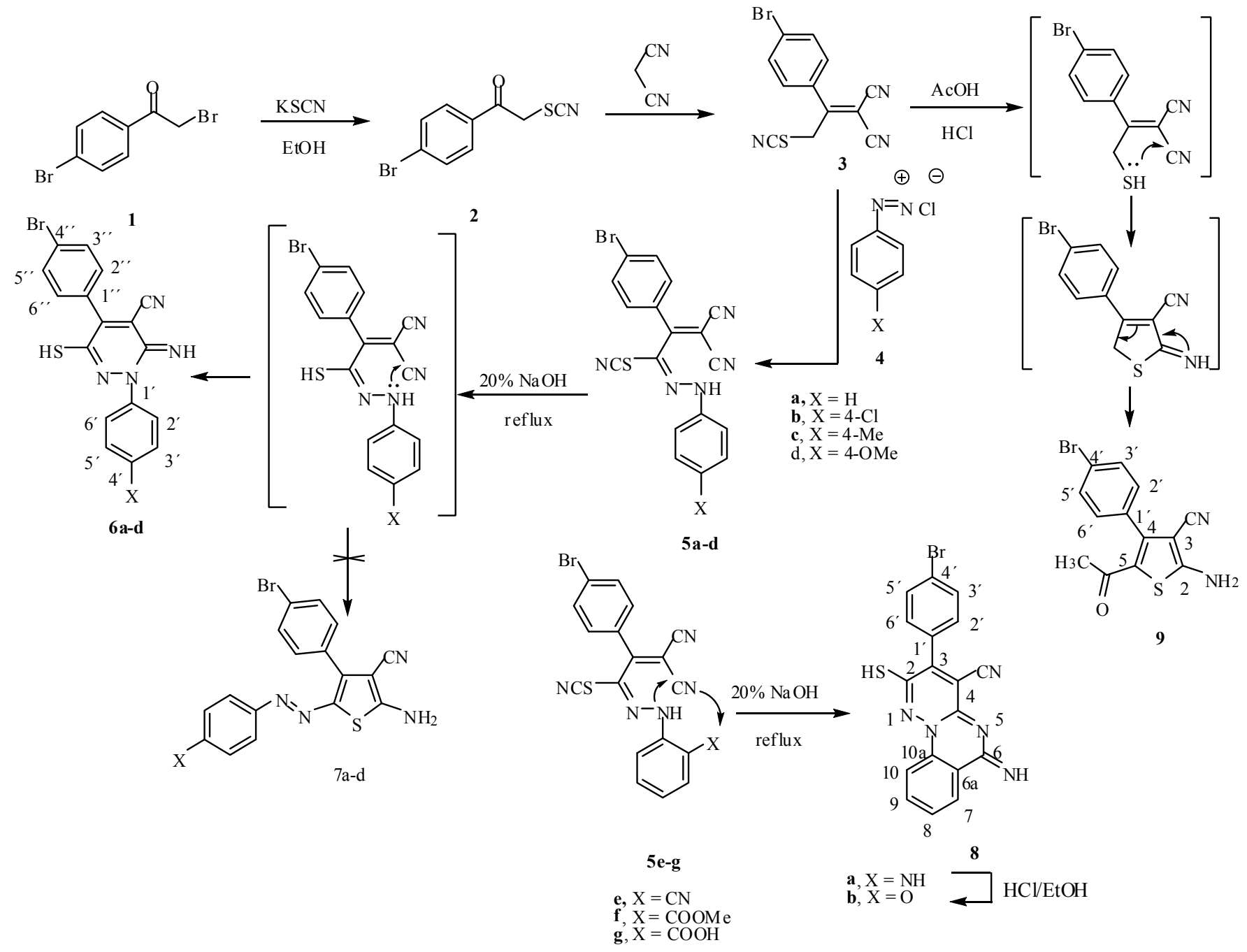

On the other hand, thiophene derivative 9 could be obtained in quantitative yield from compound 3 by refluxing in $\mathrm{AcOH} / \mathrm{HCl}$ mixture for $3 \mathrm{~h}$. The IR spectrum of 9 showed absorption bands at 3402, $2221,1680 \mathrm{~cm}^{-1}$, corresponding to $\mathrm{NH}_{2}, \mathrm{CN}$ and $\mathrm{C}=\mathrm{O}$ groups, respectively. The ${ }^{1} \mathrm{H}-\mathrm{NMR}$ spectrum of 9 revealed a singlet at $1.65 \mathrm{ppm}(3 \mathrm{H})$ and a singlet at $6.67 \mathrm{ppm}(2 \mathrm{H})$, which were attributed to the $\mathrm{CH}_{3}$ and $\mathrm{NH}_{2}$ groups, respectively, in addition to the four aromatic protons at $7.2 \mathrm{ppm}$. In the ${ }^{13} \mathrm{C}-\mathrm{NMR}$ of compound 9, 11 signals was found; the ones at 28.5, 114.4 and $186.1 \mathrm{ppm}$ were attributed to the $\mathrm{CH}_{3}$, $\mathrm{CN}$ and $\mathrm{CO}$ groups, respectively. A similar result was previously reported [18]. From these data the reaction product could be formulated as the 5-acetyl-2-aminothiophene-3-carbonitrile derivative 9. The elemental analysis of 9 was in good agreement with the proposed structure (Scheme 1).

\section{Conclusions}

Pyridazinimine derivatives 6, pyridazino[1,6-a] quinazoline $\mathbf{8}$ and 5-acetylthiophene derivative 9 have been synthesized in good yield via 2-thiocyanatoethylidene malononitrile (3). 


\section{Experimental}

\section{General}

Melting points were measured on a Gallenkamp Electrothermal melting point apparatus and are uncorrected. IR spectra (KBr pellets) were recorded on a Pye Unicam SP 3-300 Spectrophotometer. NMR spectra were recorded in DMSO- $\mathrm{d}_{6}$ on Varian Gemini 200/300 MHz NMR spectrometers using tetramethylsilane (TMS) as an internal reference. Mass spectra were registered on a Shimadzu GCMSQP 1000 Ex mass spectrometer at $70 \mathrm{eV}$. Elemental analyses were carried out at the Microanalytical Center of Cairo University.

\section{1-(4-Bromophenyl)-2-thiocyanatoethanone (2)}

To a solution of $1(10 \mathrm{mmol})$ in EtOH $(60 \mathrm{~mL})$ was added KSCN $(10 \mathrm{mmol})$. The reaction mixture was refluxed for $1.5 \mathrm{~h}$. The mixture was then poured on ice-cold water, the solid collected by filtration and recrystallized from EtOH to give yellow crystals, $80 \%$ yield, mp. $148-149^{\circ} \mathrm{C}$; IR $\left(\mathrm{cm}^{-1}\right): 2175$ (SCN), 1680 (CO); ${ }^{1} \mathrm{H}-\mathrm{NMR} \delta: 5.07$ (s, 2H, $\mathrm{CH}_{2}$ ), 7.77 (d, 2H, $\left.J=8 \mathrm{~Hz}, \mathrm{Ar}-\mathrm{H}-3^{\prime}, 5^{\prime}\right), 7.94$ (d, 2H, $J=$ $\left.8 \mathrm{~Hz}, \mathrm{Ar}-\mathrm{H}-2^{\prime}, 6^{\prime}\right) ;{ }^{13} \mathrm{C}-\mathrm{NMR} \delta: 41.56\left(\mathrm{CH}_{2}\right), 112.75(\mathrm{SCN}), 128.51\left(\mathrm{C}-4^{\prime}\right), 130.54\left(\mathrm{C}-2^{\prime}, 6^{\prime}\right), 132.03$ (C-3', 5'), $133.35\left(\mathrm{C}-1^{\prime}\right), 190.67$ (C-1); MS: $256\left(\mathrm{M}^{+}{ }^{79} \mathrm{Br}, 14 \%\right) ; 258\left(\mathrm{M}^{+}+2{ }^{81} \mathrm{Br}, 13 \%\right)$; Anal. Calcd. for $\mathrm{C}_{9} \mathrm{H}_{6} \mathrm{BrNOS}$ (256.12): C, 42.21; H, 2.36; N, 5.47. Found: C, 42.51; H, 2.65; N, 5.70.

\section{2-(1-(4-Bromophenyl)-2-thiocyanatoethylidene)malononitrile (3)}

A mixture of $2(10 \mathrm{mmol})$ and malononitrile $(10 \mathrm{mmol})$ was refluxed in $\mathrm{EtOH}(30 \mathrm{~mL})$ in the presence of piperidine $(2 \mathrm{~mL})$ for $3 \mathrm{~h}$, then left to cool at room temperature and the solid product was collected by filtration, washed with EtOH and recrystallized from EtOH to give green crystals, $74 \%$ yield, mp. $258-260^{\circ} \mathrm{C}$; IR $\left(\mathrm{cm}^{-1}\right)$ : $2210(\mathrm{CN}), 2175(\mathrm{SCN}) ;{ }^{1} \mathrm{H}-\mathrm{NMR} \delta$ : $5.41\left(\mathrm{~s}, 2 \mathrm{H}, \mathrm{CH}_{2}\right), 7.64-7.72(\mathrm{~m}$, 4H, Ar-H); MS: $304\left(\mathrm{M}^{+}{ }^{79} \mathrm{Br}, 56 \%\right) ; 306\left(\mathrm{M}^{+}+2{ }^{81} \mathrm{Br}, 53 \%\right)$; Anal. Calcd. for $\mathrm{C}_{12} \mathrm{H}_{6} \mathrm{BrN}_{3} \mathrm{~S}(304.17)$ : C, 47.38; H, 1.99; N, 13.81. Found: C, 47.67; H, 2.31; N, 14.15.

\section{General procedure for preparation of arylhydrazone derivatives $\mathbf{5} \mathbf{a}-\mathbf{g}$}

To a stirred cold solution of $3(10 \mathrm{mmol})$ and sodium acetate $(10 \mathrm{~g})$ in EtOH $(50 \mathrm{~mL})$ or pyridine $(25 \mathrm{~mL})$ was added dropwise over about 30 minutes a cold solution of a diazotized amine (aniline, 4chloro-, 4-methyl-, 4-methoxyaniline, anthranilic acid, methyl anthranilate or anthranilonitrile, 10 mmol). The stirring was continued for $1 \mathrm{~h}$ more. The coloured solids were collected by filtration, washed with cold water, and recrystallized from EtOH or 1:1 EtOH/DMF to afford 5a-g, respectively.

2-[1-(4-Bromophenyl)-2-(phenylhydrazono)-2-thiocyanatoethylidene] malononitrile (5a): Brown crystals (78\%); mp. $204-206^{\circ} \mathrm{C}$; IR $\left(\mathrm{cm}^{-1}\right)$ : 44308, $3310(\mathrm{NH}), 2214(\mathrm{CN}), 2178(\mathrm{SCN}) ;{ }^{1} \mathrm{H}-\mathrm{NMR} \delta$ : 7.34-7.60 (m, 5H, Ar-H), 7.82 (d, 2H, $J=8 \mathrm{~Hz}, \mathrm{Ar}-\mathrm{H}), 7.95$ (d, 2H, $J=8 \mathrm{~Hz}, \mathrm{Ar}-\mathrm{H}), 9.90$ (s, 1H, NH).; 
MS: $408\left(\mathrm{M}^{+},{ }^{79} \mathrm{Br}, 38 \%\right) ; 410\left(\mathrm{M}^{+}+2,{ }^{81} \mathrm{Br}, 35 \%\right)$; Anal. Calcd. for $\mathrm{C}_{18} \mathrm{H}_{10} \mathrm{BrN}_{5} \mathrm{~S}$ (408.28): C, 52.95; H, 2.47; N, 17.15. Found: C, 53.23; H, 2.71; N, 16.98 .

2-[1-(4-Bromophenyl)-2-[(4-chlorophenyl)hydrazono]-2-thiocyanatoethylidene] malononitrile (5b): Yellow solid (81\%); mp. 210-212 ${ }^{\circ} \mathrm{C}$; IR $\left(\mathrm{cm}^{-1}\right)$ : 4388, $3330(\mathrm{NH}), 2218(\mathrm{CN}), 2175$ (SCN); ${ }^{1} \mathrm{H}-\mathrm{NMR}$

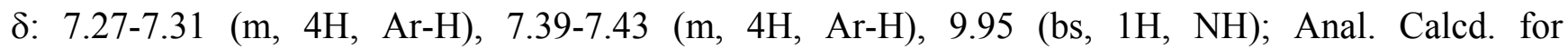
$\mathrm{C}_{18} \mathrm{H}_{9} \mathrm{BrClN}_{5} \mathrm{~S}$ (442.72): C, 48.83; H, 2.05; N, 15.82. Found: C, 49.12; H, 2.11; N, 16.17.

2-[1-(4-Bromophenyl)-2-thiocyanato-2-(p-tolylhydrazono)ethylidene] malononitrile (5c): Yellow solid (74\%); mp. 218-219 ${ }^{\circ} \mathrm{C}$; IR (cm $\left.{ }^{-1}\right):$ 4395, $3318(\mathrm{NH}), 2221(\mathrm{CN}), 2177(\mathrm{SCN}) ;{ }^{1} \mathrm{H}-\mathrm{NMR}$ 8: 2.27 (s, 3H, $\left.\mathrm{CH}_{3}\right), 7.10(\mathrm{~d}, 2 \mathrm{H}, J=9 \mathrm{~Hz}, \mathrm{Ar}-\mathrm{H}), 7.47$ (d, 2H, $\left.J=9 \mathrm{~Hz}, \mathrm{Ar}-\mathrm{H}\right), 7.64$ (d, 2H, $\left.J=8 \mathrm{~Hz}, \mathrm{Ar}-\mathrm{H}\right), 7.92(\mathrm{~d}$, $2 \mathrm{H}, J=8 \mathrm{~Hz}, \mathrm{Ar}-\mathrm{H}), 9.65$ (s, $1 \mathrm{H}, \mathrm{NH}$ ); Anal. Calcd. for $\mathrm{C}_{19} \mathrm{H}_{12} \mathrm{BrN}_{5} \mathrm{~S}$ (422.30): C, 54.04; H, 2.86; N, 16.58. Found: C, 54.34; H, 3.12; N, 16.44.

2-[1-(4-Bromophenyl)-2-[(4-methoxyphenyl)hydrazono]-2-thiocyanatoethylidene] malononitrile (5d): Yellow solid (74\%); mp. 214-216 ${ }^{\circ} \mathrm{C}$; IR $\left(\mathrm{cm}^{-1}\right)$ : 4395, $3325(\mathrm{NH}), 2224(\mathrm{CN}), 2174$ (SCN); ${ }^{1} \mathrm{H}-\mathrm{NMR}$

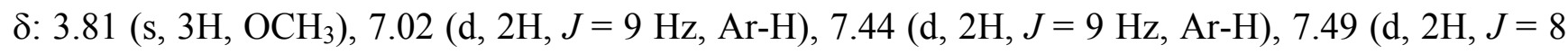
$\mathrm{Hz}, \mathrm{Ar}-\mathrm{H}), 7.91$ (d, 2H, $J=8 \mathrm{~Hz}, \mathrm{Ar}-\mathrm{H}), 11.05$ (s, 1H, NH); Anal. Calcd. for $\mathrm{C}_{19} \mathrm{H}_{12} \mathrm{BrN}_{5} \mathrm{OS}(438.30)$ : C, 52.07; H, 2.76; N, 15.98. Found: C, 52.37; H, 3.03; N, 16.24.

2-[1-(4-Bromophenyl)-2-[(2-cyanophenyl)hydrazono]-2-thiocyanatoethylidene] malononitrile (5e): Yellow solid (71\%); mp. 205-207 ${ }^{\circ} \mathrm{C}$; IR $\left(\mathrm{cm}^{-1}\right)$ : 4385, $3330(\mathrm{NH}), 2220,2212(\mathrm{CN}), 2177(\mathrm{SCN}) ;{ }^{1} \mathrm{H}-$ NMR $\delta$ : 7.49-7.65 (m, 4H, Ar-H), 7.74-8.30 (m, 4H, Ar-H), 10.95 (s, 1H, NH); Anal. Calcd. for $\mathrm{C}_{19} \mathrm{H}_{9} \mathrm{BrN}_{6} \mathrm{~S}$ (433.29): C, 52.67; H, 2.09; N, 19.40. Found: C, 52.39; H, 2.33; N, 19.24.

2-[N'-[2-(4-Bromophenyl)-3,3-dicyano-1-thiocyanatoethylidene] hydrazono]benzoic acid methyl ester (5f): Yellow solid (77\%); mp. 199-201 ${ }^{\circ} \mathrm{C}$; IR ( $\left.\mathrm{cm}^{-1}\right)$ : 3439, $3310(\mathrm{NH}), 2210(\mathrm{CN}), 2175(\mathrm{SCN}), 1668$ (CO) $\mathrm{cm}^{-1}$; ${ }^{1} \mathrm{H}-\mathrm{NMR} \delta: 2.62\left(\mathrm{~s}, 3 \mathrm{H}, \mathrm{CH}_{3}\right), 7.27-7.49$ (m, 4H, Ar-H), 7.65-8.30 (m, 4H, Ar-H), 11.10 (s, $1 \mathrm{H}, \mathrm{NH})$. MS: $465\left(\mathrm{M}^{+}-1,{ }^{79} \mathrm{Br}, 31 \%\right) ; 467\left(\mathrm{M}^{+}+1,{ }^{81} \mathrm{Br}, 32 \%\right)$; Anal. Calcd. for $\mathrm{C}_{20} \mathrm{H}_{12} \mathrm{BrN}_{5} \mathrm{O}_{2} \mathrm{~S}$ (466.31): C, 51.51; H, 2.59; N, 15.02. Found: C, 51.72; H, 2.71; N, 15.30.

2-[ N'-[2-(4-bromophenyl)-3,3-dicyano-1-thiocyanatoallylidene]hydrazino)benzoic acid (5g): Yellow solid (70\%); mp. 225-227 ${ }^{\circ}$; IR ( $\left.\mathrm{cm}^{-1}\right)$ : 3414, $3335(\mathrm{NH}), 2214(\mathrm{CN}), 2178(\mathrm{SCN}), 1685(\mathrm{CO}) ;{ }^{1} \mathrm{H}-$ NMR 8: 7.09-7.49 (m, 4H, Ar-H), 7.65-7.98 (m, 4H, Ar-H), 10.66 (s, 1H, NH), 14.03 (s, 1H, COOH); Anal. Calcd. for $\mathrm{C}_{19} \mathrm{H}_{10} \mathrm{BrN}_{5} \mathrm{O}_{2} \mathrm{~S}$ (452.29): C, 50.46; H, 2.23; N, 15.48. Found: C, 50.81; H, 2.11; N, 15.61 .

\section{General procedure for preparation of compounds $\mathbf{6 a - d}$ and $\mathbf{8 a}, \mathbf{b}$}

To a solution of each of 5a-g $(10 \mathrm{mmol})$ in $\mathrm{EtOH}(25 \mathrm{~mL})$ was added $20 \%$ aqueous $\mathrm{NaOH}$ solution $(10 \mathrm{~mL})$. The reaction mixture was refluxed for $2 \mathrm{~h}$, then left to cool. The precipitated solid products 
formed were collected by filtration, washed with cold water, and recyrstallized from EtOH or 1:1 EtOH/DMF to afford 6a-d and $\mathbf{8 a}, \mathbf{b}$ respectively.

5-(4-Bromophenyl)-3-imino-6-mercapto-2-phenyl-2,3-dihydropyridazine-4-carbonitrile (6a): Brown crystals (70\%), mp. 233-235 ${ }^{\circ} \mathrm{C}$; IR ( $\left.\mathrm{cm}^{-1}\right): 3404,3318(\mathrm{NH}), 2206(\mathrm{CN}) ;{ }^{1} \mathrm{H}-\mathrm{NMR} \delta: 6.09$ (s, 1H, SH), 7.14-7.42 (m, 2H, Ar-H), 7.52-7.60 (m, 3H, Ar-H), 7.75 (d, 2H, $J=8 \mathrm{~Hz}, \operatorname{Ar}-\mathrm{H}), 8.17$ (d, 2H, $J=8 \mathrm{~Hz}$, $\mathrm{Ar}-\mathrm{H}), 8.33$ (s, 1H, NH); MS: $383\left(\mathrm{M}^{+},{ }^{79} \mathrm{Br}, 19 \%\right)$; $385\left(\mathrm{M}^{+}+2,{ }^{81} \mathrm{Br}, 18 \%\right)$; Anal. Calcd. for $\mathrm{C}_{17} \mathrm{H}_{11} \mathrm{BrN}_{4} \mathrm{~S}$ (383.27): C, 53.27; H, 2.89; N, 14.62. Found: C, 53.33; H, 2.73; N, 14.53.

5-(4-Bromophenyl)-2-(4-chlorophenyl)-3-imino-6-mercapto-2,3-dihydropyridazine-4-carbonitrile (6b): Brown crystals (64\%), mp. 263-265 ${ }^{\circ} \mathrm{C}$; IR $\left(\mathrm{cm}^{-1}\right)$ : 3400, $3322(\mathrm{NH}), 2209(\mathrm{CN})$; ${ }^{1} \mathrm{H}-\mathrm{NMR}$ 8: 6.45 (s, 1H, SH), 7.54 (d, 2H, J=8 Hz, Ar-H), 7.67 (d, 2H, $J=8$ Hz, Ar-H), 7.89-8.10 (m, 4H, Ar-H), 8.29 (s, $1 \mathrm{H}, \mathrm{NH}$ ); Anal. Calcd. for $\mathrm{C}_{17} \mathrm{H}_{10} \mathrm{BrClN}_{4} \mathrm{~S}$ (417.71): C, 48.88; H, 2.41; N, 13.41. Found: C, 48.69; H, $2.54 ; \mathrm{N}, 13.77$.

5-(4-Bromophenyl)-3-imino-6-mercapto-2-p-tolyl-2,3-dihydropyridazine-4-carbonitrile (6c): Brown crystals (64\%), mp. 245-247 ${ }^{\circ}$; IR $\left(\mathrm{cm}^{-1}\right)$ : 3390, $3332(\mathrm{NH}), 2212(\mathrm{CN})$; ${ }^{1} \mathrm{H}-\mathrm{NMR}$ 8: $2.34(\mathrm{~s}, 3 \mathrm{H}$, $\mathrm{CH}_{3}$ ), 5.87 (s, 1H, SH), 7.24 (d, 2H, $\left.J=9 \mathrm{~Hz}, \mathrm{Ar}-\mathrm{H}\right), 7.44$ (d, 2H, $\left.J=9 \mathrm{~Hz}, \mathrm{Ar}-\mathrm{H}\right), 7.61$ (d, 2H, $J=9$ $\mathrm{Hz}, \mathrm{Ar}-\mathrm{H}), 8.26$ (d, $2 \mathrm{H}, J=9 \mathrm{~Hz}, \mathrm{Ar}-\mathrm{H}), 8.39$ (s, 1H, NH); Anal. Calcd. for $\mathrm{C}_{18} \mathrm{H}_{13} \mathrm{BrN}_{4} \mathrm{~S}$ (397.29): C, 54.42; H, 3.30; N, 14.10. Found: C, 54.75; H, 3.56; N, 14.34 .

5-(4-bromophenyl)-3-imino-6-mercapto-2-(4-methoxyphenyl)-2,3dihydropyridazine-4-carbonitrile (6d): Brown crystals (69\%), mp. 249-251 ${ }^{\circ} \mathrm{C}$; IR (cm $\left.{ }^{-1}\right): 3395,3320(\mathrm{NH}), 2208(\mathrm{CN}) ;{ }^{1} \mathrm{H}-\mathrm{NMR} \delta: 3.65$ $\left(\mathrm{s}, 3 \mathrm{H}, \mathrm{OCH}_{3}\right), 6.80(\mathrm{~s}, 1 \mathrm{H}, \mathrm{SH}), 7.26-7.87(\mathrm{~m}, 8 \mathrm{H}, \mathrm{Ar}-\mathrm{H}), 8.27(\mathrm{~s}, 1 \mathrm{H}, \mathrm{NH}) ;{ }^{13} \mathrm{C}-\mathrm{NMR} \delta: 55.48$ $\left(\mathrm{OCH}_{3}\right), 89.81(\mathrm{C}-4), 112.43(\mathrm{CN}), 114.83\left(\mathrm{C}-3^{\prime} 5^{\prime}\right), 115.93\left(\mathrm{C}-4^{\prime \prime}\right), 118.59\left(\mathrm{C}-2^{\prime}, 6^{\prime}\right), 126.20(\mathrm{C}-$ 2"',6"), 129.84 (C-3"',5'), 132.08 (C-1"'), 141.88 (C-1'), 148.97 (C-6), 155.42 (C-4'), 159.76 (C-5), 161.46 (C-3); Anal. Calcd. for $\mathrm{C}_{18} \mathrm{H}_{13} \mathrm{BrN}_{4} \mathrm{OS}$ (413.29): C, 52.31; H, 3.17; N, 13.56. Found: C, 52.45; H, 3.28; N, 13.84 .

3-(4-Bromophenyl)-6-imino-2-mercapto-6H-pyridazino[1,6-a]quinazoline-4-carbonitrile (8a): Dark brown solid (59\%), mp. 314-316 ${ }^{\circ} \mathrm{C}$; IR $\left(\mathrm{cm}^{-1}\right): 3435(\mathrm{NH}), 2224(\mathrm{CN})$; ${ }^{1} \mathrm{H}-\mathrm{NMR} \delta: 6.88(\mathrm{~s}, 1 \mathrm{H}, \mathrm{SH})$, 7.20 (d, 1H, $J=8 \mathrm{~Hz}, \mathrm{Ar}-\mathrm{H} 10), 7.55-7.58$ (m, 1H, Ar-H8), 7.68 (d, 1H, J=8 Hz, Ar-H7), 7.77 (d, 2H, $\left.J=9 \mathrm{~Hz}, \mathrm{Ar}-\mathrm{H}, 2^{\prime}, 6^{\prime}\right), 7.96-7.99$ (m, 1H, Ar-H9), 8.24 (d, 2H, $\left.J=9 \mathrm{~Hz}, \mathrm{Ar}-\mathrm{H}, 3^{\prime}, 5^{\prime}\right), 8.31$ (s, 1H, $\mathrm{NH}) ;{ }^{13} \mathrm{C}-\mathrm{NMR} \delta$ : $105.46(\mathrm{C}-4), 116.20(\mathrm{CN}), 121.06$ (C-10), 122.08 (C-6a), 125.53 (C-4'), 126.50 (C7), 126.58 (C-2', 6'), 128.97 (C-8), 129.98 (C-3', 5'), 135.64 (C-9), 140.90 (C-1'), 141.22 (C-10a), 150.97 (C-2), 155.80 (C-3), 156.42 (C-4a), 161.95 (C-6); Anal. Calcd. for $\mathrm{C}_{18} \mathrm{H}_{10} \mathrm{BrN}_{5} \mathrm{~S}$ (408.27): C, 52.95; H, 2.47; N, 17.15. Found: C, 52.79; H, 2.69; N, 17.34 .

3-(4-Bromophenyl)-2-mercapto-6-oxo-6H-pyridazino[1,6-a]quinazoline-4-carbonitrile (8b): Violet crystals (77\%), mp. $277-279^{\circ} \mathrm{C}$; IR $\left(\mathrm{cm}^{-1}\right): 2214(\mathrm{CN}), 1674(\mathrm{C}=\mathrm{O}) ;{ }^{1} \mathrm{H}-\mathrm{NMR} \delta: 6.80(\mathrm{~s}, 1 \mathrm{H}, \mathrm{SH})$, 7.61-7.66 (m, 1H, Ar-H10), 7.81-7.87 (m, 1H, Ar-H8), 7.91-7.99 (m, 1H, Ar-H9), 8.09 (d, 2H, $J=8$ 
$\left.\mathrm{Hz}, \mathrm{Ar}-\mathrm{H}, 2^{\prime}, 6^{\prime}\right), 8.20-8.31$ (m, 1H, Ar-H7), 8.86 (d, 2H, $J=9 \mathrm{~Hz}$, Ar-H 3',5'); Anal. Calcd. for $\mathrm{C}_{18} \mathrm{H}_{9} \mathrm{BrN}_{4} \mathrm{OS}$ (409.26): C, 52.83; H, 2.22; N, 13.69. Found: C, 53.12; H, 2.44; N, 14.04.

\section{Transformation of $\mathbf{8 a}$ into $\mathbf{8 b}$ (General procedure)}

To a solution of $8 \mathbf{a}(50 \mathrm{mmol})$ in ethanol $(25 \mathrm{~mL})$ was added concentrated $\mathrm{HCl}(5 \mathrm{~mL})$ and the mixture was refluxed for $1 \mathrm{~h}$. After cooling to room temperature, the reaction mixture was diluted with cold water and neutralized with ammonia. The solid formed were collected by filtration and recrystalized from EtOH/DMF (1:1) to afford products identical in all respects (mp, mixed mp and TLC) with $\mathbf{8 b}$.

\section{5-Acetyl-2-amino-4-(4-bromophenyl)thiophene-3-carbonitrile (9)}

To a solution of $3(50 \mathrm{mmol})$ in acetic acid $(25 \mathrm{~mL})$ was added concentrated $\mathrm{HCl}(10 \mathrm{~mL})$ and the mixture was refluxed for $3 \mathrm{~h}$. After cooling to room temperature, the reaction mixture was diluted with cold water and neutralized with ammonia. The solid formed was collected by filtration and recrystalized from EtOH/DMF (1:1) to afford 9. Yellow crystals $(65 \%), \mathrm{mp} .237-239^{\circ} \mathrm{C}$; IR $\left(\mathrm{cm}^{-1}\right)$ : $3402\left(\mathrm{NH}_{2}\right), 2221(\mathrm{CN}), 1680(\mathrm{C}=\mathrm{O}) ;{ }^{1} \mathrm{H}-\mathrm{NMR} \delta: 1.65\left(\mathrm{~s}, 3 \mathrm{H}, \mathrm{CH}_{3}\right), 6.67\left(\mathrm{~s}, 2 \mathrm{H}, \mathrm{NH}_{2}\right), 7.08(\mathrm{~d}, 2 \mathrm{H}, J$ $=9 \mathrm{~Hz}, \mathrm{Ar}-\mathrm{H}), 7.39$ (d, 2H, $J=9 \mathrm{~Hz}, \mathrm{Ar}-\mathrm{H}) ;{ }^{13} \mathrm{C}-\mathrm{NMR} \delta: 28.54\left(\mathrm{CH}_{3}\right), 88.59(\mathrm{C}-3), 114.47\left(\mathrm{C}-3^{\prime}, 5^{\prime}\right)$, $114.68(\mathrm{CN}), 118.20$ (C-4'), 127.92 (C-2',6'), 132.21 (C-5), 135.20 (C-1'), 147.45 (C-2), 159.37 (C4), 186.15 (CO); MS: $321\left(\mathrm{M}^{+}\right)$; Anal. Calcd. for $\mathrm{C}_{13} \mathrm{H}_{9} \mathrm{BrN}_{2} \mathrm{OS}$ (321.19): C, 48.61; H, 2.82; N, 8.72. Found: C, 48.87; H, 3.12; N, 8.89.

\section{References}

1. Oka, Y.; Omura, K.; Miyake, A.; Itoh, K.; Tomimoto, M.; Tada, N.; Yurugi, S. Studies on the Syntheses of N-Heterocyclic Compounds. XXV. Syntheses of Pyrido[3,4-d]pyridazine Derivatives. Chem. Pharm. Bull. 1975, 23, 2239-2250 .

2. Oka, Y.; Omura, K.; Miyake, A.; Itoh, K.; Tomimoto, M.; Tada, N.; Yurugi, S. Studies on synthesis of N-Heterocyclic compounds XXV. Synthesis of pyrido[3,4-d]pyridazine derivatives 2. Chem. Pharm. Bull. 1975, 23, 1488-1499.

3. Matsuura, I.; Yoneda, F.; Nitta, Y. Pyridazines. XI. Pyrido[2, 3-d]pyridazines. II. Chem. Pharm. Bull. 1966, 14, 1010-1016.

4. Tigler, M.; Stanovnik, B. In Condensed Pyridazines Including Cinnolines and Phthalazines; Castle, R. N. Ed.; John Wiley \& Sons, Inc.: New York, NY, USA, 1973; pp. 968-1012.

5. Matyus, P. 3(2H)-Pyridazinones: Some Recent Aspects of Synthetic and Medicinal Chemistry. $J$. Heterocycl. Chem. 1998, 35, 1075-1089.

6. Mylari, B.L.; Zembrowski, W.J. Pyrido[2,3-d]pyridazinones as aldose reductase inhibitors. US Pat. 4,954,629, 1990; [Chem. Abstr. 1991, 114, 62105u].

7. Barrett, J.A..; Woltmann, R.F.; Swillo, R.S.; Kasiewski, C. Pharmacology of RG W-2938: a cardiotonic agent with vasodilator activity. J. Cardiovasc. Pharm. 1990, 16, 537-45. 
8. Hewett, R.; Pettit, S. N.; Smith, P. Pyrido [2,3-d] pyridazine derivatives as herbicides. Can. Pat. Appl. CA 2,086,898, 1993; [Chem. Abstr. 1994, 121, 9415p].

9. Sahin, M. F.; Badiçoglu, B.; Gökçe, M.; Küpeli, E.; Yesilada, E. Synthesis and analgesic and antiinflammatory activity of methyl 6-substituted-3(2H)-pyridazinone-2-ylacetate derivatives. Arch Pharm (Weinheim) 2004, 337, 445-452.

10. Frank, H.; Heinisch, G. Pharmacologically Active Pyridazines Part I. In Progress in Medicinal Chemistry; Ellis, G.P.; West, G. B., Eds.; Elsevier: Amsterdam, The Netherlands, 1990; 27, p. 149.

11. Frank, H.; Heinisch, G. Pharmacologically Active Pyridazines Part II. In Progress in Medicinal Chemistry, Ellis, G.P., Luscombe, D.K., Eds.; Elsevier: Amsterdam, The Netherlands, 1992; 29, p. 141-183.

12. Al-Sheikh, M. A.; Medrassi, H. Y.; Elnagdi M. H.; Hafez, E. A. Substituted hydrazonals as building blocks in heterocyclic synthesis: a new route to arylhydrazonocinnolines. J. Chem. Res. 2007, 432-436.

13. Al-Sheikh, M.A.; Salaheldin, A.M.; Hafez, E.A.; Elnagdi, M.H. 2-Arylhydrazono-3-oxopropanals in Heterocyclic synthesis: Synthesis of arylazopyrazole, arylazoisoxazole and dialkylpyridazine5,6-dicarboxylate Derivatives. New one-step synthesis of arylazopyrimidines. J. Heterocycl. Chem. 2004, 41, 647-654.

14. Al-Sheikh, M. A. ; Salaheldin, A.M.; Elnagdi, M. H.; Hafez, E. A. $\alpha$-Enones in heterocyclic synthesis, Part I. Classical synthetic and environmentally friendly synthetic approaches to alkyl and acyl azoles and azines. J. Chem. Res. 2004, 174.

15. Hafez, E.A.; Khalifa, M. A. E.S.; Guda, K.; Elnagdi, M. H. Reactions with the arylhydrazones of a-cyanoketones: Utility of arylhydrazonomesoxalonitriles for the synthesis of azoles and azolines. Z. Naturforsch. 1980, 35b, 485.

16. Gewald, K.; Hain, U. Substituted 2-aminopyrroles and 2-hydroxypyrroles from ylidene nitriles. Synthesis 1984, 62.

17. Elnagdi, M. H. ; Sadek, K. U.; Taha, N. M.; Yassin, Y. M. The structure of products of coupling of arenediazonium salts with 3-aminocrotononitrile derivatives. Collect. Czech. Chem. Commun. 1990, $55,734$.

18. Abderazek, F. M. Heterocyclic synthesis with nitriles: A novel synthesis of some thiophene and thieno[2,3-d]pyrimidine derivatives. Z. Naturforsch. 1989, 44b, 488.

Sample availability: Contact the author.

(C) 2008 by the authors; licensee Molecular Diversity Preservation International, Basel, Switzerland. This article is an open-access article distributed under the terms and conditions of the Creative Commons Attribution license (http://creativecommons.org/licenses/by/3.0/). 